\title{
SOBRE LA LEGITIMIDAD DE LA OPINIÓN PÚBLICA COMO SUSTENTO DE LA POLÍTICA PENAL
}

\author{
Rafael Velandia Montes \\ Universidad La Gran Colombia
}

\section{Resumen}

Hoy en día son frecuentes propuestas de reformas normativas de carácter penal que sostienen ser la respuesta a los reclamos de la mayoría de la ciudadanía. En el presente planteamiento se afirma que una gran parte de la sociedad exige, sobre todo, la ampliación del campo de acción del Derecho Penal por medio de la criminalización de diversas conductas generadoras de conflictos sociales y el aumento de penas para los comportamientos que ya se encuentran tipificados como delito. En este trabajo se hace una consideración sobre la legitimidad de dichas invocaciones en las propuestas de cambio legislativo penal.

Palabras clave: reforma jurídica, crimen, opinión pública, Derecho Penal.

El autor: abogado, especialista en Ciencias Penales y Criminológicas, diploma en Estudios Avanzados en el área de Filosofía del Derecho y doctor en Sociología Jurídica e Instituciones Políticas. Dirección postal: calle 140 No 10A-36 Interior 1, apartamento 302.E-mail: rafaelvelandiamontes@hotmail.com

Recibido: 2 de febrero de 2014; evaluado: 13 de abril de 2014; aceptado: 27 de mayo de 2014 . 


\title{
ON THE LEGITIMACY OF PUBLIC OPINION AS SUPPORT OF PENAL POLICY
}

\author{
Rafael Velandia Montes \\ Universidad La Gran Colombia
}

\begin{abstract}
At the present time, proposals of penal normative reforms that argue they are the answer to the claims of the majority of citizens are frequent. Under this approach, it has been stated that a large part of society demands, mainly, the increase of the Criminal Law's field of action through the criminalization of different conducts triggering social conflicts, and the rise of penalties for the behaviors already typified as offenses. In this way, the purpose of this paper is to reflect on the legitimacy of such statements in the proposals for criminal law change.
\end{abstract}

Keywords: Legal reform, crime, public opinion, Criminal law

About the author: Lawyer specialized in Penal Science and Criminology with a Certificate Program in Advanced Studies in the area of Philosophy of Law. Doctor in Legal Sociology and Political Institutions. Address: Calle 140 No 10A-36 Interior 1, apt.302.E-mail: rafaelvelandiamontes@hotmail.com

Received: February 2, 2014; reviewed: April 13, 2014; accepted: May 27, 2014. 


\section{Reclamos sociales y política penal: ${ }^{1}$ el Derecho Penal como único recurso}

Hoy en día, en los proyectos de modificación legislativa penal es frecuente tomar en cuenta la opinión pública o los deseos de la sociedad. Lo anterior puede verse en el caso colombiano, en el cual se han empleado dichas reivindicaciones, bien para aumentar las sanciones que pueden imponerse para determinados comportamientos, como en la delincuencia sexual ${ }^{2}$ o frente a conductas en el ámbito de la seguridad vial. ${ }^{3}$ De tal manera, quienes los promueven sostienen que tales cambios normativos de naturaleza penal, que consisten en la criminalización de diversas acciones generadoras de conflictos sociales y en el aumento de penas para los comportamientos que ya se encuentran tipificados como delito, no son más que el acatamiento de los deseos de la opinión pública. Hay dos afirmaciones principales en este tipo de argumentación: la primera es que las propuestas de reforma normativa son la respuesta a los deseos de la opinión pública; la segunda, que los deseos de esta son siempre de maximización del Derecho Penal.

A continuación nos ocuparemos de dichos argumentos. Empero, antes de continuar, debemos aclarar que no se pretende hacer una investigación sobre invocaciones de tal tipo en casos concretos; de lo que se trata aquí es de analizar la legitimidad de aquellas invocaciones a la hora de plantear propuestas de reforma legislativa en el área penal. Pasamos, entonces, a discurrir al respecto.

1 Se prefiere el uso de la expresión "política penal" en lugar de "política criminal", porque el uso de este término en lugar de aquel "corresponde a la visión que percibe a la criminalidad como una realidad empírica, con propiedades ontológicas, por ende, habría un objeto de conocimiento con atributos o de naturaleza criminal, cuyo estudio informaría la política respectiva", pero "la definición de criminal es producto de un proceso político de criminalización, un acto de definición subjetiva el cual emerge de juicios de valor, no una cualidad que corresponda a la descripción del mundo objetivo". Asimismo, "la política en este terreno no tiene como receptores únicos a los sujetos ni a las situaciones rotuladas como criminales, puesto que la política mencionada" también "está destinada al conjunto de la población, afectada o comprometida por sus medidas o intervenciones". Por último, "la política criminal es una expresión arcaica frente al derecho, pues hace décadas fue sustituida la nominación de derecho criminal por la de derecho penal, más acorde con su objeto y finalidades". Germán Silva García, Criminología. Teoría sociológica del delito (Bogotá: Instituto Latinoamericano de Altos Estudios (ILAE), 2011), 5 y 46.

2 Rafael Velandia Montes, "Delincuencia sexual y populismo penal en Colombia", URVIO, Revista Latinoamericana de Seguridad Ciudadana, núm. 11 (marzo 2012): 26 y ss.

3 Rafael Velandia Montes, "Inseguridad vial y política penal en Colombia", Derecho Penal Contemporáneo-Revista Internacional, núm. 45 (octubre-diciembre 2013): 123 y ss. 


\subsection{Democracia directa frente a democracia representativa}

El primer argumento que se utiliza con frecuencia como fundamento de proyectos de reforma normativa de carácter penal ${ }^{4}$ consiste en que los cambios propuestos son la respuesta a los deseos de la opinión pública. Esta afirmación debe ser analizada en relación con los fundamentos de la democracia, pues en ella la participación del público es un elemento esencial. En una democracia o, como se entiende contemporáneamente, una república, ${ }^{5}$ el tamaño actual de las sociedades imposibilita que los ciudadanos intervengan de manera directa en la toma de las decisiones correspondientes a un Estado en materia legislativa; ${ }^{6}$ por eso, dicha participación del público tiene lugar de dos formas: mediante democracia directa o representativa. No se trata acá de abordar esta cuestión con profundidad, sino de brindar una sucinta diferenciación al respecto. De acuerdo con Mezey, en oposición a una democracia directa, una democracia representativa se caracteriza porque: 1) "la política pública es hecha por los representantes de la gente y no por la gente misma"; 2) "los representantes son elegidos por los ciudadanos"; 3) "todos los ciudadanos adultos, con solo algunas raras excepciones, tienen permitido votar por representantes y todos los ciudadanos tienen un voto" y 4) "los representantes son responsables por sus acciones ante aquellos que los eligieron [...] y pueden ser reemplazados por ellos en las siguientes elecciones".

En la democracia directa son los ciudadanos los que intervienen de manera inmediata en la toma de decisiones. Así, por ejemplo, en el caso colombiano, la Ley 134 de 1994 establece la iniciativa popular y el referendo aprobatorio o derogatorio ${ }^{8}$

4 Con ello no se afirma que solo ocurra en el ámbito penal, sino que solo nos ocupamos de esta área del Derecho. Las conclusiones son extrapolables a las demás áreas, aunque debe reconocerse que este tipo de invocaciones se aprecia con mayor claridad y frecuencia en el Derecho Penal.

5 Por exceder la materia de este escrito, no podemos ocuparnos de si la democracia existe o no. Sin embargo, cabe señalar que se asume una posición en sentido negativo. Al respecto, puede verse Jhon Gastil, By Popular Demand. Revitalizing Representative Democracy through Deliberative Elections (Londres: University of California Press, 2000), 10 y ss. De otro lado, Mezey pone de presente que los sistemas representativos que predominan en la actualidad "no son democráticos porque el autogobierno, que es lo que significa la democracia, no es la misma cosa que gobierno por alguien más, incluso si se elige a ese alguien para gobernar en su nombre". Michael L. Mezey, Representative Democracy Legislators and their Constituents (Lanham: Rowman \& Littlefield Publishers, Inc., 2008), 17.

6 Es evidente que no es el único asunto de competencia estatal, pero solo haremos mención a esta área, ya que en este escrito nos ocupamos de las decisiones en materia legislativa y, de manera aún más específica, de naturaleza penal.

7 Mezey, Representative Democracy Legislators and their Constituents, 17.

8 Aunque en esta ley también se establecen la revocatoria de mandato, el plebiscito y la consulta popular como mecanismos de participación ciudadana, solo mencionamos la iniciativa popular legislativa y el referendo aprobatorio o derogatorio, debido a que son las herramientas que pueden involucrar una reforma normativa de naturaleza penal. 
como sus mecanismos. Sin embargo, nuestra reflexión no trata sobre el ejercicio de alguno de los mecanismos de participación ciudadana de la democracia directa que tienen la posibilidad de generar cambios normativos penales, sino acerca del planteamiento, por parte de quienes fungen como representantes en la democracia representativa, de modificaciones en el ámbito penal que, se supone, tienen sustento en los deseos de la opinión pública.

Lo primero que debe señalarse es que:

[...] partimos de una concepción en la que la opinión pública no existe y que es un instrumento empleado por los detentadores del poder, con el propósito de ayudar a disminuir la eventual resistencia social que pueda existir frente a la adopción de una medida política, legislativa, económica o social dirigida a hacer frente a un conflicto social, haciendo creer que existe un consenso general respecto de su implementación. ${ }^{9}$

Empero, también es cierto que a "pesar de que no aceptamos la existencia de la opinión pública, reconocemos que su mención en las sociedades actuales en diversos escenarios es recurrente, hecho que no puede pasarse por alto y que nos obliga a ocuparnos de ella", ${ }^{10}$ mención que se ha hecho en el caso colombiano en cuanto a la delincuencia sexual ${ }^{11}$ y a la seguridad vial, por ejemplo. ${ }^{12}$

A partir de la argumentación de los representantes respecto a estar cumpliendo los deseos de la opinión pública, lo que debe preguntarse es cuál es el papel de los representantes si, de acuerdo con su manifestación, solo sería necesario hacer encuestas de opinión pública e implementar lo que se supone solicitado en ellas. De cualquier manera, bajo ninguna circunstancia se puede aceptar que:

[...] una reforma normativa penal se fundamente en lo que la ciudadanía aparentemente quiere o no según encuestas públicas: no se trata de desconocer la participación de los ciudadanos en la toma de decisiones que les afecten, pues la democracia participativa con sus diversos instrumentos hace posible tal intervención, sino de rechazar la tesis de que los resultados de un sondeo

Velandia Montes, "Inseguridad vial y política penal en Colombia", 128.

Velandia Montes, "Inseguridad vial y política penal en Colombia", 128.

Velandia Montes, "Delincuencia sexual y populismo penal en Colombia", 26 y ss.

Velandia Montes, "Inseguridad vial y política penal en Colombia", 122 y ss. 
son prueba definitiva e irrefutable de los deseos de la ciudadanía frente a una reforma legislativa, en este caso, penal. ${ }^{13}$

Tampoco se está afirmando que "exista un sondeo modelo o un valor ideal o normativo de encuestas que permita afirmar que con x número de sondeos sí se puede conocer lo que la sociedad desea", ${ }^{14}$ porque lo cierto es que son muy conocidas sus limitaciones, la posibilidad de influenciar sus respuestas y lo que estas dicen. ${ }^{15}$

Si la sociedad o parte de ella desea un cambio normativo real, el camino a seguir sería utilizar alguno de los mecanismos de participación ciudadana que estén establecidos en los respectivos ordenamientos nacionales, ${ }^{16}$ lo cual, por supuesto, no sería obstáculo para que los representantes elegidos, en ejercicio de sus competencias, también pudieran plantear propuestas similares o idénticas a las hechas por los representados en ejercicio de los postulados de la democracia directa, según se ha explicado. Aquí no se sostiene que los representantes no puedan encarnar la opinión de sus representados, porque una aseveración en tal sentido llevaría a la inexplicable e ilógica afirmación de que los representantes serían elegidos no por ser portadores de ideas comunes a los representados, sino por motivos ajenos a ello. Por lo contrario, se trata de que los representantes deben incorporar mesura, racionalidad, objetividad y cientificidad en la toma de decisiones que involucren cambios normativos, todo en aras del bienestar social. Empero, se ha observado que los mencionados factores no están presentes cuando es la ciudadanía o parte de ella la que asume el ejercicio de la competencia de hacer propuestas de cambio normativo, pues lo que se busca es la satisfacción de intereses diversos que no apuntan a la solución verdadera del conflicto social en cuestión. ${ }^{17}$

13 Velandia Montes, "Inseguridad vial y política penal en Colombia", 138-139.

14 Velandia Montes, "Inseguridad vial y política penal en Colombia", 138-139.

15 Velandia Montes, "Delincuencia sexual y populismo penal en Colombia", 26 y ss; Velandia Montes, "Inseguridad vial y política penal en Colombia", 122.

16 En el caso colombiano, aquellos establecidos en la Ley 134 de 1994. De todas maneras, no parece deseable que la política penal se determine mediante la iniciativa popular legislativa y el referendo aprobatorio o derogatorio, pues la política penal debe estar fundamentada en investigación criminológica y no en otro tipo de sustentos de tipo emocional, como ocurrió en el caso de la Ley 1327 de 2009. Velandia Montes, "Delincuencia sexual y populismo penal en Colombia", 26 y ss. La afirmación sobre los fundamentos de la política penal se hace desde el entendido de que la Sociología Jurídica Penal es una "subespecialidad de la sociología jurídica" que "se dedica al estudio de la divergencia de interés penal y de las instituciones relativas al control social penal que pretende ejercerse sobre ella, con la finalidad de definir la política penal". Silva García, Criminología, 12.

17 Como se evidenció en el caso de la delincuencia sexual y la Ley 1327 de 2009. Velandia Montes, "Delincuencia sexual y populismo penal en Colombia", 26 y ss. 
En este momento es necesario recordar las palabras proferidas por el expresidente estadounidense James Madison ${ }^{18}$ hace más de 220 años, cuando señalaba dos puntos importantes que diferencian la democracia y la república. Apuntaba que la primera consistía en que en esta, a diferencia de aquella, había delegación del gobierno en un pequeño número de ciudadanos elegidos por el resto, ${ }^{19}$ lo que tenía como efecto:

[...] refinar y ampliar la opinión pública pasándola por el medio de un cuerpo de ciudadanos elegidos, cuya sabiduría quizá pueda discernir mejor el verdadero interés de su país y cuyo patriotismo y amor por la justicia quizá haga menos probable sacrificarlo por consideraciones temporales o parciales. ${ }^{20}$

Desde tal entendido, también podía pasar que "la voz pública, pronunciada por los representantes de la gente [fuera] más consonante con el bien público que si fuera pronunciada por la misma gente convocada para tal propósito". ${ }^{21}$

Por ende, el papel de un representante acucioso, diligente y responsable es pasar por los filtros de la mesura, racionalidad, objetividad y cientificidad las ideas que

18 James Madison, "The Same Subject Continued: The Union as a Safeguard against Domestic Faction and Insurrection" en The Federalist Papers, ed. J. R. Pole (Indianápolis: Hackett Publishing Company, Inc., 2005), 52.

19 La segunda diferencia consistía en el mayor número de ciudadanos y en la mayor esfera del país sobre los que la república podía extenderse. Madison, "The Same Subject Continued", 52.

20 Madison, sin embargo, había advertido que podría ocurrir el efecto contrario: "Hombres de temperamentos facciosos, o con prejuicios locales o con designios siniestros, quizá, por intriga, corrupción o por otras razones, puedan primero obtener los sufragios y entonces traicionar los intereses de la gente". Ante tal consideración, Madison se preguntaba si una república pequeña o una grande era la mejor para elegir "guardianes apropiados de la prosperidad pública" y tomaba partido por una república grande por dos razones: la primera era que, sin importar cuán pequeña fuera la república, los representantes debían ser elevados a cierto número "para protegerla de la conspiración de unos pocos" y, sin considerar cuán grande fuera, los representantes debían ser limitados "a cierto número para protegerla de la confusión de la multitud". Por lo tanto, "del número de representantes en los dos casos, no siendo proporcional a sus representados, y siendo más proporcional en la república pequeña, se sigue que si la proporción de personas adecuadas no es menor en la república grande que en la pequeña en aquella se presentará una mayor opción y, consecuentemente, una mayor probabilidad de una elección adecuada". En segundo lugar, puesto que "cada representante será elegido por un mayor número de ciudadanos en una república grande que en una pequeña, será más difícil para candidatos indignos practicar con éxito artes inmorales, con las cuales las elecciones son frecuentemente llevadas a cabo; y los sufragios de las personas serán más libres, será más probable que se centren en hombres que posean los méritos más atractivos y los caracteres más prolijos y establecidos". Madison también advierte que en todo caso se encontrarían inconvenientes tanto en la república grande como en la pequeña, porque, al aumentar el número de electores, se ocasionaría que los representantes sean poco conocedores de "todas las circunstancias locales y de los intereses menores" y, si se disminuyera mucho el número de electores, se ataría indebidamente a los representantes a dichas circunstancias e intereses y se les haría poco aptos para comprender y perseguir grandes objetivos nacionales. Madison, "The Same Subject Continued", 52-53.

21 Madison, "The Same Subject Continued", 52-53. 
puedan llegar por parte de los representados, así como las propias, con el fin de determinar la decisión normativa más adecuada para el bienestar social, al margen del área jurídica de la que se trate, aunque aquí nos centramos en el ámbito penal. En esta línea, no es defendible para un representante, salvo una perspectiva de absoluta irresponsabilidad en relación con sus deberes y de indiferencia hacia la ciencia, sostener que las propuestas de reforma normativa penal que propone no deben ser más que el reflejo, sin ningún filtro, de la voluntad de la opinión pública.

Asimismo, no puede pasarse por alto que es necesario darle mayor protagonismo a la educación en el ámbito legal a los ciudadanos, porque solo ella tiene la capacidad de concienciarlos sobre la importancia y las consecuencias que una iniciativa popular legislativa — legal o constitucional_ o un referendo puede tener para el conglomerado social y que las decisiones de política pública, entre ellas, la política penal, no pueden ser tomadas con base en aspectos emocionales, como respuesta a deseos de las víctimas de delitos, de sus familiares, amigos o personas que se sientan identificadas con ellas, al margen del motivo de dicha identificación. En la misma línea, cabe señalar que los medios de comunicación deben asumir con mayor responsabilidad su tarea y brindar a la ciudadanía información proporcionada sobre los conflictos sociales relevantes, para evitar crear la sensación de que un conflicto social es de mayor gravedad de la que tiene; ${ }^{22}$ este tipo de actuación sirve de sustento predominante, si no exclusivo, en los casos de procesos de iniciativa popular legislativa en materia penal.

En definitiva, a modo de resumen, sobre los fundamentos de la democracia representativa, no es justificable para los representantes basar proposiciones de reforma normativa con el argumento de estar cumpliendo los deseos de los representados por dos razones: en primer lugar, porque no puede afirmarse con certeza que los deseos de la sociedad puedan ser conocidos mediante sondeos de opinión pública ${ }^{23}$ y, en segundo lugar, porque, incluso aunque se aceptara la posibilidad de conocerlos de tal manera, corresponde a los representantes ejercer con diligencia su cargo, lo cual significa que deben tener en cuenta factores de mesura, racionalidad, objetividad y cientificidad a la hora de determinar el contenido de las propuestas de cambio normativo, así estas correspondan a los supuestos deseos de la opinión pública o

22 Dicho proceso de satanización se puede ver en el caso de la seguridad vial en Colombia. Velandia Montes, "Inseguridad vial y política penal en Colombia", 132 y ss.

23 La problemática determinación de los deseos de la opinión pública mediante encuestas de opinión pública puede verse, por ejemplo, en materia de delincuencia sexual. Velandia Montes, "Delincuencia sexual y populismo penal en Colombia", 26 y ss. y de seguridad vial Velandia Montes, "Inseguridad vial y política penal en Colombia", 136 y ss. 
de un sector de ella. A continuación estudiaremos otro elemento que caracteriza a muchas de las propuestas contemporáneas de reforma preceptiva que se asumen como la respuesta a los deseos de la opinión pública: el Derecho Penal como único mecanismo para hacer frente a los diversos conflictos sociales existentes.

\subsection{El Derecho Penal como prima ratio}

Una vez expuesto el porqué es irrazonable que los legisladores sostengan que las propuestas de reforma normativa penal no deben ser más que el reflejo, sin ningún filtro, de la voluntad de la opinión pública, corresponde ahora examinar un aspecto característico de dichas propuestas: el uso exclusivo del Derecho Penal para hacer frente a cualquier conflicto social. Si bien "actualmente existe un relativo consenso sobre el papel del Derecho Penal como herramienta para enfrentar conflictos sociales graves, siempre y cuando los recursos con los que cuenta el ordenamiento en sus demás ramas sean ineficaces para darles solución", ${ }^{24}$ que es como "se entiende al principio de subsidiariedad o ultima ratio, característico del Derecho Penal de un Estado democrático y que fija límites al poder estatal sancionatorio debido a que aquel posee las sanciones más drásticas, representadas en restricción a la libertad, que el Estado puede imponer a los ciudadanos", ${ }^{25}$ lo cierto es que este principio es pasado por alto en las propuestas de reforma normativa penal a las que hemos venido refiriéndonos, pues ellas se centran en lo penal y ni siquiera exponen de modo sucinto por qué otras herramientas jurídicas distintas a la penal o extrajurídicas no son idóneas para hacer frente al conflicto social del que se trate. En tales proyectos de reforma normativa penal, lo que brilla por su ausencia es una explicación razonable, objetiva y científica de por qué se debe recurrir directamente al Derecho Penal y por qué no hay otro medio que posibilite afrontar un conflicto social. En dichos proyectos se evidencia una falta de investigación criminológica que sirva de sustento a tal postura, así como una conversión del principio de ultima ratio del Derecho Penal, que ahora pasar a ser prima ratio y sin justificación, se reitera, es proclamado como herramienta idónea de primer orden. Este carácter de prima ratio que le ha sido atribuido materialmente al Derecho Penal en oposición a lo que, de forma dogmática, se le imputa como ultima ratio, plantea problemas en relación con los límites del Derecho Penal. El interrogante que surge es ¿existen o no límites a las propuestas de reforma normativa que hemos venido examinando? Ese aspecto será tratado a continuación.

Velandia Montes, "Delincuencia sexual y populismo penal en Colombia", 20.

Velandia Montes, "Delincuencia sexual y populismo penal en Colombia", 20. 


\section{Política penal y reclamos sociales: ¿cambios sin límites?}

Si el Derecho Penal ha perdido en gran parte su carácter de ultima ratio, que ha sido transformado en prima ratio, cabe preguntarse, entonces, si hay o no límites a su campo de acción. Debe apuntarse que sí existen límites a las propuestas de reforma normativa penal, aun en el caso de aquellas de iniciativa popular, que están representadas en las normas imperativas de Derecho Internacional y en los convenios internacionales de derechos humanos. Para dar unos ejemplos, ${ }^{26}$ si se tiene en cuenta que la pena de muerte y la cadena perpetua ${ }^{27}$ son medidas que se mencionan como respuesta a supuestas demandas de la opinión pública frente a los delincuentes, debe indicarse que en Colombia la institución de la pena de muerte no es posible jurídicamente, como quiera que mediante la Ley 297 de 1996 se aprobó el Segundo Protocolo Facultativo del Pacto Internacional de Derechos Civiles y Políticos, destinado a abolir la pena de muerte, adoptado por la Asamblea General de las Naciones Unidas el 15 de diciembre de 1989, en cuyo Artículo 1 se prohíbe la ejecución de cualquier persona sometida a la jurisdicción de un Estado parte. En lo que respecta a la cadena perpetua, si se formulara una propuesta de acto legislativo, ${ }^{28}$ incluso por la vía del referendo, sería necesario esperar una decisión al respecto por parte de la Corte Constitucional sobre si un cambio en tal sentido equivale o no a una sustitución de la Constitución Política ${ }^{29}$ y, por ende, inaceptable y solo posible en el evento de que fuera aprobado por una Asamblea Constituyente.

26 No es posible emprender un análisis de las diversas medidas que se han planteado en materia penal.

27 Como se hizo en la Ley 1327 de 2009. Velandia Montes, "Delincuencia sexual y populismo penal en Colombia", 21 y ss.

28 El Artículo 34 de la Constitución Política la prohíbe.

29 Si bien la Ley 1327 de 2009, que convocaba a referendo constitucional para decidir sobre si se podía o no permitir la imposición de la cadena perpetua en la comisión de determinados delitos, fue declarada inconstitucional en la Sentencia C-397 de 2010 de la Corte Constitucional, la declaratoria fue solo por vicios durante el trámite del proyecto de ley ante el Congreso de la República. El primer vicio fue que no se adjuntó "la certificación del Registrador Nacional del Estado Civil, prevista en los artículos 24 y 27 de la Ley Estatutaria de Mecanismos de Participación Ciudadana —-Ley 134 de 1994—. Específicamente la certificación de manera completa sobre cumplimiento de los requisitos constitucionales y legales. La ausencia de esta certificación inhibe la iniciación del trámite legislativo y vicia la constitucionalidad de todo el procedimiento adelantado ante el Congreso de la República. Este es un vicio que afecta la validez del trámite legislativo al resultar un elemento fundamental en la legitimación del proyecto de iniciativa ciudadana que salvaguarda la democracia y los mecanismos de participación ciudadana". El segundo vicio consistió en "la modificación sustancial del texto original del proyecto de ley respaldado por la iniciativa ciudadana (principio de identidad flexible). En primer término, se modificó la expresión 'procederá la pena de prisión perpetua de acuerdo con la ley' por la frase 'se podrá imponer hasta la pena de prisión perpetua, de acuerdo con la ley'; para la Corte, la sustancialidad del cambio consiste en que mientras la primera expresión preveía en forma imperativa la imposición de la prisión perpetua, la segunda lo hacía facultativamente. Adicionalmente, se cambiaron las expresiones 'maltrato severo' por 'lesiones personales agravadas' y se agregó un nuevo parágrafo a nivel de política de prevención y constitucionalización de una institución como el Instituto Colombiano de Bienestar Familiar". Además, la Corte Constitucional señaló 
Con independencia de las indeseables consecuencias jurídicas de las propuestas de reforma normativa penal a las que hemos hecho mención —en tanto retroceso en afectación de derechos de los ciudadanos- y desde el entendido de que tienen como fin la adopción de medidas crueles, desproporcionadas y que no persiguen ningún propósito real de solución del conflicto social al que manifiestan hacer frente, un problema adicional de este tipo de planteos es que puede generar la percepción social de que el camino está despejado para hacer modificaciones normativas penales de cualquier índole; esto, a su vez, causaría más actitudes sociales de aprobación a cualquier clase de modificación normativa penal y propuestas ciudadanas adicionales en tal sentido, sin que importe cuán atroces o inhumanas sean, situación indeseable en un Estado moderno. Si se asume que el sistema jurídico colombiano permite tanto la reforma legal como la constitucional mediante iniciativa popular, ${ }^{30}$ es de especial importancia el papel que los legisladores tienen, según se mencionó, de ejercer con diligencia su cargo, lo cual significa que deben considerar factores de mesura, racionalidad, objetividad y cientificidad a la hora de fijar el contenido de las propuestas de cambio normativo, así sean los supuestos deseos de la sociedad o de un sector de ella, pues los planteamientos que hacen, sean o no aprobados, son de conocimiento público y pueden provocar la percepción de que todo vale y de que el fuego se combate con fuego, una línea de argumentación irracional e indeseable.

\section{Conclusiones}

- Las decisiones de política pública, entre las que está la política penal, deben obedecer al interés general y no al individual; dicho interés en el campo penal está fijado por la adopción de medidas de este tipo solo cuando no haya herramientas extrajurídicas o jurídicas distintas para afrontar un conflicto social grave.

- La política penal debe estar fundamentada en investigación criminológica, pues por medio de esta se puede establecer de modo adecuado cuándo las herramientas extrajurídicas o jurídicas distintas a la penal tienen la capacidad de hacer frente a un conflicto social de naturaleza grave o, en su defecto, cuándo es necesaria la intervención del Derecho Penal, todo en aras del respeto de su principio de ultima ratio.

que no revisó los vicios de competencia. Colombia, Corte Constitucional, Sentencia C-397 de 25 de mayo de 2010, M. P. Juan Carlos Henao Pérez.

30 No se desconoce, imprueba, descalifica, censura o rechaza la iniciativa popular, pero sí se advierte que debe obrarse con especial precaución cuando se recurre a este mecanismo de participación ciudadana, mucho más si se trata de medidas que involucran la afectación de los derechos fundamentales de las personas. No nos oponemos a que las personas participen en la toma de las decisiones que las afectan, sino a que se tomen decisiones de carácter normativo sin fundamento científico. 
3) La invocación de la opinión pública como sustento de propuestas de reformas de carácter penal aparece como irrazonable y carece de legitimidad en democracias representativas, en primer lugar, porque la determinación de lo que se quiere por parte de la opinión pública no es algo que se pueda determinar con certeza, ni siquiera con encuestas, debido a los diversos problemas de representatividad y sesgo que ellas tienen. En segundo lugar, porque, aunque se pudiera conocer la voluntad de los representados, los representantes deben incorporar mesura, racionalidad, objetividad y cientificidad en la toma de decisiones que involucren cambios normativos penales, todo en aras del bienestar social. Dicho de otra forma, las propuestas de reforma normativa penal de los representantes deben involucrar mesura, racionalidad, objetividad y cientificidad, que solo pueden obtenerse mediante la investigación criminológica y que no están presentes en los planteamientos derivados de los supuestos deseos de la opinión pública, que se sustentan en intereses individuales de diversos tipos y que se caracterizan por su emotividad y naturaleza draconiana.

\section{Referencias}

Colombia, Corte Constitucional. Sentencia C-397 de 25 de mayo de 2010. M. P. Juan Carlos Henao Pérez.

Gastil, Jhon. By Popular Demand. Revitalizing Representative Democracy through Deliberative Elections. Londres: University of California Press, 2000.

Madison, James. "The Same Subject Continued: The Union as a Safeguard against Domestic Faction and Insurrection" en The Federalist Papers, editado por J. R. Pole, 246-307. Indianápolis: Hackett Publishing Company, Inc., 2005.

Mezey, Michael L. Representative Democracy Legislators and their Constituents. Lanham: Rowman \& Littlefield Publishers, Inc., 2008.

Silva García, Germán. Criminología. Teoría sociológica del delito. Bogotá: Instituto Latinoamericano de Altos Estudios (ILAE), 2011.

Velandia Montes, Rafael. "Delincuencia sexual y populismo penal en Colombia”. URVIO, Revista Latinoamericana de Seguridad Ciudadana, núm. 11 (marzo 2012): 19-32.

Velandia Montes, Rafael. "Inseguridad vial y política penal en Colombia". Derecho Penal Contemporáneo-Revista Internacional, núm. 45 (octubre-diciembre 2013): 119-158. 